\title{
Application of neural network technologies for inflation forecasting
}

\author{
Dadyan E.G. \\ Department of data analysis, decision-making and financial technologies \\ Financial University under the Government of the Russian Federation \\ Moscow, Russian Federation \\ dadyan60@yandex.ru
}

\begin{abstract}
The paper considers the methodology of forecasting the level of inflation in Russia with the help of analytical platform Deductor Studio. In solving the problem, the mechanisms of data purification from noises and anomalies were applied, which ensured the quality of forecast model construction and receipt of forecast values for five months in advance. The principle of forecasting the time series was also demonstrated: import, seasonal detection, cleaning, smoothing, construction of forecast model, and forecasting the inflation rate for five months ahead.
\end{abstract}

Keywords- Deductor Studio, forecasting of time series, forecasting of inflation rate, construction of forecast model, construction of forecast.

\section{INTRODUCTION}

Inflation is one of the indicators of macroeconomic stability and a factor on which the socio-economic development of the country depends to a large extent. Forecasting inflation for appropriate decision-making to maintain a favorable economy level is an important task for any country, including the Russian Federation. Forecasting inflation for appropriate decision-making to maintain a favorable economy level is an important task for any country, including the Russian Federation. Forecasting is one of the most demanded tasks of Business Analytics. And this is not surprising: knowing, even with a certain error, the nature of events in the future, you can make more informed management decisions, plan activities, develop appropriate sets of measures, efficiently allocate resources, etc. From the view point of data analysis technologies, prediction can be considered as the definition of some unknown value by a set of related values. Therefore, prediction is done using Data Mining tasks such as regression, classification, and clustering. The paper provides a methodology for analyzing the dependence of inflation on the time factor for several years in the Russian Federation in order to predict this important factor, in the end.

\section{GENERAL SCHEME OF CONSTRUCTION OF ANALYTICAL SOLUTION FOR INFLATION FORECASTING}

The paper considers the methodology of forecasting the level of inflation in Russia with the help of analytical platform Deductor Studio. Deductor Studio has in its arsenal the necessary and versatile means to solve the problem of product sales forecasting, which allow:

- to perform graphical analysis of time series;
- $\quad$ analyze the series for the presence of trend and seasonality;

- $\quad$ preprocessing time Series - remove anomalies and smooth out;

- build different predictive models and evaluate their accuracy and adequacy;

- $\quad$ Predict and execute postprocessing forecast values (once grouping, analytical reporting, export forecast to Data warehouse or other source, etc.).Prepare Your Paper Before Styling

III. SEARCH AND FORMATION OF THE TABLE OF INITIAL DATA. IMPORT OF INITIAL DATA "INFLATION RATE IN RUSSIA" IN THE DATABASE DEDUCTOR. TO GENERATE THE ORIGINAL

\section{CHART}

The actual data on inflation calculated on the base of indices of considers Price in the Russian Federation, are publisher by the Several Services of State Statistics (http://finplus.ru/ru/info/inflation_index/Russia) in the form of the corresponding table.

This data on inflation in Russia for the period from 2006 to 2017 and three months of 2018 are used in the work. Each cell in the table you use indicates how many percent of the price has changed for the corresponding period. The price reduction is shown with a minus sign. I would like to emphasize once again that inflation is calculated on the basis of the index of consumer prices for goods and services, officially published by the Federal Service of State Statistics of the Russian Federation.

Data import is the starting point for analyzing data. Import to Deductor can be done from popular data storage formats such as Excel, Access, MS SQL, Oracle, text file and others. In addition, you have universal access to any data source through $\mathrm{ADO}$ or ODBC. Import is done by calling the Import Wizard in the Scripts panel of the Deductor Studio analytic platform. Deductor Studio.

At the end of this step the initial diagram is formed using the visualizer - the graph of dependence of the inflation level on time is drawn.

Because the inflation rate was calculated monthly, you must convert the Date field to the date (year + month) View this operation is performed using the date and time handler 


\section{OPERATION «AUTOCORRELATION»: DETECTING SEASONALITY}

To analyze the data from the point of view of seasonality of the inflation rate in Russia we will use autocorrelation method. Autocorrelation is a correlation (relationship) between a value and its delay in one or more periods of time. Such values are calculated sequentially for each lag period, and a graph of autocorrelation coefficients for various lag in time (autocorrelation function, ACF) is plotted.

By ACF graph, you can determine whether the data is random, whether the data is trend and seasonality, whether the data is stationary.

In Deductor Studio to evaluate the kind of ACF (autocorrelation function) is the autocorrelation handler. To determine the annual seasonality, the number of counts in the case of monthly data is recommended [1] to put at least 12-13. If the data series is a case (or there is a nonlinear trend), the autocorrelation factors for any lag will be close to zero. If a series has a linear trend, the neighboring values of YT and YT1 have a strong correlation, and autocorrelation coefficients are significantly different from zero for the first several lag periods, and with the increase of the period gradually decrease to zero. The autocorrelation factor for lag time equal to one period is often very large (close to 1). The autocorrelation factor for lag time equal to two periods will also be large. However, it will not be as large as the lag time in one period. If a series has a seasonal component, a significant autocorrelation coefficient will be observed for lag periods equal to or multiple of the seasonal period. The seasonal lag period is 12 for monthly data.

\section{PARTIAL PREPROCESSING: REMOVAL OF ANOMALIES AND SMOOTHING. SPECTRAL TREATMENT: WAVELET TRANSFORMATION}

Partial preprocessing is designed to convert time series to remove uncharacteristic bursts and fluctuations. An anomaly is a sharp deviation of the value from its expected value ("outliers" and "Falling"). The reasons for this may be different, and, as a rule, they are of force majeure: The panic mood of the population, changes in legislation, etc. To obtain a qualitative prediction of the anomaly must be edited. To do this, the Deductor has a special tool in the partial processing handler. In it the editing of anomalies is made on the basis of algorithm of robust filtration. There are three decrease of suppression (small, medium, high), which characterizes the measure of tolerance to the size of permissible emissions.

Spectral processing is intended for transformation of time series with the purpose of removal of high-frequency or seasonal oscillations from them. Anti-aliasing and filtration in Deductor is understood as the application of mathematical methods of digital signal processing to the time series for the purpose of restoring the initial signal against the background of interference, determining the availability of a useful signal, etc. these algorithms Embedded in the "partial processing" handler of the Deductor analytical platform.

The smoothing, as well as the editing of anomalies, should be treated cautiously. Anomalies can be of a nonrandom nature, i.e. bursts become statistically significant. In addition, smoothing to some extent idealizes the overall picture of the time series. Therefore, specific recommendations on smoothing to give difficult, it depends on the data of the time series. As recommended in [1] on the basis of the accumulated experience of modeling such systems, when processing the original curve, do not set the value of the "bandwidth" parameter too low, optimally 50-80.

After work of the processor "partial processing" and performance of spectral processing data faded, the trend became visible.

\section{CONVERTING DATA TO A SLIDING WINDOW}

When solving the problems of prediction of time series using a neural network, it is necessary to submit to the analyzer input the values of several, contiguous, counts from the initial dataset. This method of data selection is called a sliding window [2] (window - because only some contiguous section of data is allocated, sliding - because this window "moves" over the complete set). In this case, the efficiency of implementation is noticeably increased if you do not select data each time from several consecutive records, and sequentially arrange data related to a window position in one record.

The values in one of the record fields will refer to the current count, while in others they are offset from the current count to the future or to the past. Thus, the transformation of the sliding window has two parameters: "Depth of immersion"the number of "past" counts, falling into the window, and "forecast Horizon"-the number of "future" counts.

It should be noted that for the boundary (relative to the beginning and the end of the whole sample) the window positions will be formed incomplete records, i.e. records containing empty values for missing past or future counts. The conversion algorithm allows to exclude such records from the sample (then for several boundary records will not be formed), or to include them (then formed records for all the available counts, but some of them will be incomplete). To properly form a sliding window, the data must be ordered accordingly.

To transform our data, we choose the Deductor analytical Platform engine called sliding window. When setting important parameters of the handler, it is necessary to consider that the seasonality was detected earlier (item IV). Therefore, the inflation forecast model should be based on data for 12 and 11 months ago, a month ago and the current month. Based on the above, it is also necessary to select the depth of immersion 12 and forecast Horizon 1.

In the end, the sliding window engine generates the required data set to build the inflation forecast.

Now you can set the task to run the inflation forecast, for example, 5 months in advance.

VII. ENTERING THE DATA OF THE SLIDING WINDOW INTO THE NEURAL NETWORK. FORMATION OF THE TRAINING SAMPLE. NEURAL NETWORK TRAINING 
Before you use network, you need to train it. The task of training here is tantamount to the task of approximation of a function, that is to restore a function by its separate points - a table-defined function. Thus, for training it is necessary to prepare a table with input values and corresponding to them output values, that is to prepare a tutorial sample. In our case, the training sample is a table of the results of the sliding window handler. Next, the parameters defining the structure of the neural network-the number of hidden layers and neurons in them, as well as the activation function of neurons are set. Many authors [1-5] argue that when choosing the number of neurons should be guided by the following rule: "The number of connections between neurons should be much less than the number of examples in the training set." The number of links is calculated as the relationship of each neuron to all the neurons of adjacent layers, including the connections on the input and output layers. Too many neurons can lead to the so-called "retraining" of the network, when it yields good results on the examples included in the training sample, but practically does not work on other examples. After configuring your network configuration, you should choose an algorithm for learning. As the same authors point out, neural networks using the reverse propagation algorithm are a powerful tool for searching for patterns, forecasting and qualitative analysis. To build a prediction in our case we open the "Processing Wizard" and select "Neural Network" in it. Then set the necessary input and output factors:

\section{Setting the column assignment,}

2. Defining the structure of the neural network,

3. Selecting the algorithm and setting the training parameters,

4. Start the neural network learning process.

As a result of the training, you can observe the change in values and the final indicators of average and maximum errors, as well as the percentage of recognized examples. The resulting scatter chart clearly shows the quality of the training.

\section{FORECASTING THE PROCESS USING THE PREDICTION WIZARD}

After training the neural network, it remains only to build the required forecast of the inflation rate in Russia. Forecasting allows you to predict the values of inflation by the number of counts corresponding to a given forecast horizon. To configure the prediction algorithm, you set the forecast Horizon and the table fields that you want to submit to the model input to build the prediction (to calculate the output field of the model). In the Deductor analytical platform, you must use the Prediction Processing Wizard to configure its parameters. Initially, there is an important question: what can be the maximum forecast horizon? The following rule is recommended [1]: The number of historical data should be 10-15 times greater than the forecast horizon. This means that if the length of a series of more than 100 counts (data for 17 years) the maximum forecast horizon may well be 5 months. The forecasting wizard is used to forecast the inflation rate in the Russian Federation in the following sequence:
1. Set up a column relationship to predict the time series, set the forecast horizon;

\section{Determination of the display method;}

3. Set up different parameters of the forecast chart columns.

After the special visualizer "forecast chart" is launched, the forecast of the inflation rate in the Russian Federation is displayed for five months ahead (Fig. 1). The larger portion of the same chart is shown below in Figure 2.

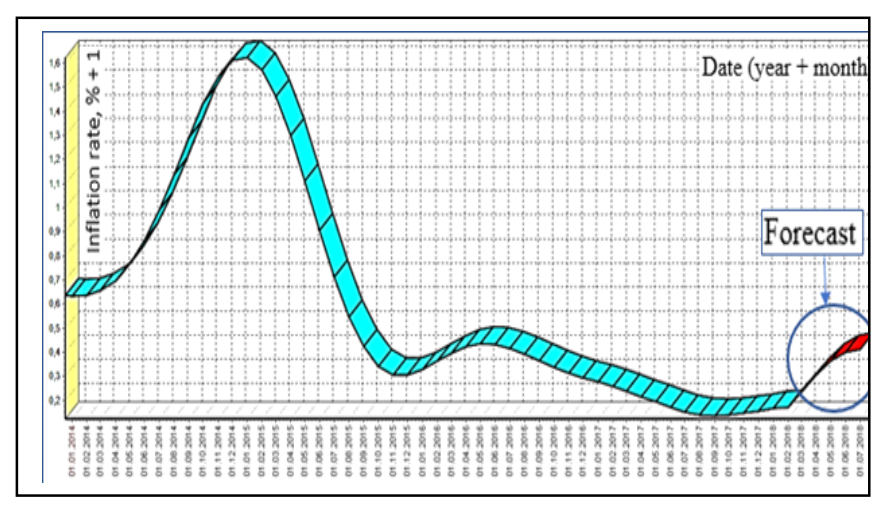

Figure 1. Forecast of inflation rate in Russia for five months ahead

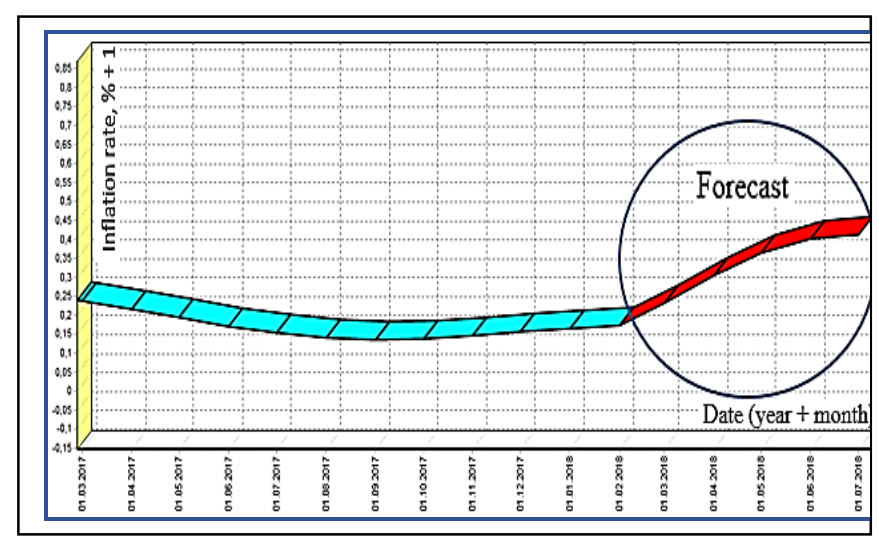

Figure 2. Increased forecast of inflation rate in Russia for five months ahead

\section{CONCLUSION}

The paper considers the methodology of forecasting the level of inflation in Russia with the help of analytical platform Deductor Studio. In solving the problem, the mechanisms of data purification from noises and anomalies were applied, which ensured the quality of forecast model construction and receipt of forecast values for five months in advance. The principle of forecasting the time series was also demonstrated: import, seasonal detection, cleaning, smoothing, construction of forecast model, and forecasting the inflation rate for five months ahead.

Such a methodology is the basis of any prediction of the time series with the difference that for each case it is necessary 
both to get the necessary time series and to choose the parameters of data cleansing, forecast model parameters.

Interesting results in our opinion, confirming the correctness of the presented methodology - practical coincidence of the forecast skill of inflation in the Russian Federation (Fig. 16) with the data of the Federal Service of State statistics on 1.04.2018.

\section{REFERENCES}

[1] Paclín N., Oreshkov O. (business intelligence: from data to knowledge, BaseGroup Labs, LLC «leader», 20014, Saint-Petersburg.

[2] Barras, L., O. Scaillet, et al. False Discoveries in Mutual Fund Performance: Measuring Luck in Estimated Alphas. The Journal of Finance - 2010 - 65(1) - c. 179-216.

[3] Brown, K. C. and F. K. Reilly. Analysis of investments and management of portfolios, 2009, South-Western Cengage Learning

[4] Daniel, K., M. Grinblatt, et al. "Measuring Mutual Fund Performance with Characteristic-Based Benchmarks". The Journal of Finance - 1997 - 52(3) - c. 1035-1058

[5] Elton, E. J., M. J. Gruber. (2003). Incentive Fees and Mutual Funds. The Journal of Finance -58(2) - c. 779-804

[6] Dadyan E.G. Foreign exchange market "turbulence the economy." Problems of modern science and education/Problems of modern science and education, 2014, №12 (30).
[7] Dadyan E.G. Foreign exchange market Russia during a deep economic crisis. Collection of scientific works of 15 international scientificpractical Conference "new information technologies in education" ("1C" technology to form a innovation education and business Wednesday)Publishing, 2015, Moscow.

[8] Dadyan E.G. (Influence of some factors on the formation of foreign exchange rates. V International Conference "Science in modern information society» 26-01/27/15, North Charleston, USA (IV). Vol. 2. SPC Academic. Create Space 4900 LaCross Road. North Charleston, SC 29406, 2015, USA.

[9] Dadyan E.G. (Influence of some factors on the formation of foreign exchange rates. Fundamental and applied science today. Fundamental and applied sciences today IV. Vol. 2. SPC Academic. Create Space 4900 LaCross Road. North Charleston, SC 29406, 2014, USA, pp. 233240.

[10] Dadyan E.G. Prediction of effectiveness of investments in mutual funds. Academic science-challenges and achievements. Academic science-problems and achievements (IV). Vol. 3. North Charleston. 2014, USA, p. 244-251.

[11] Dadyan E.G. A system for forecasting the efficiency of investments in mutual funds as application $1 \mathrm{c}$ Enterprise. Latest information technologies. Proceedings of the fourteenth international scientificpractical Conference «adopting technologies " $1 \mathrm{c}$ " to improve the effectiveness of education organizations "-Publishing, 2014, Moscow, p. 344-197.

[12] Dadyan E.G., Configuration and simulation in the system "1C: Enterprise 8 ". The collection of scientific works of the 18th International Scientific-Practical Conference "current information technologies in education". Under the commonly. Red. D.V. Chistov. 2018. S. 200-203. 